\title{
Inflammation- and ischemia-induced shedding of venular glycocalyx
}

\author{
A. W. Mulivor and H. H. Lipowsky \\ Department of Bioengineering, Pennsylvania State University, University Park, Pennsylvania 16802
}

Submitted 2 September 2003; accepted in final form 23 December 2003

\begin{abstract}
Mulivor, A. W., and H. H. Lipowsky. Inflammation- and ischemia-induced shedding of venular glycocalyx. Am J Physiol Heart Circ Physiol 286: H1672-H1680, 2004. First published January 2, 2004; 10.1152/ajpheart.00832.2003.-Alterations in the composition of the glycocalyx of venular endothelium in postcapillary venules (rat mesentery) were explored in models of inflammation and ischemia-reperfusion injury. Lectins were covalently linked to fluorescently labeled microspheres $(0.1-\mu \mathrm{m}$ diameter) or directly labeled with FITC. Adhesion of lectins specific for glucose and galactose residues of glycosaminoglycans (GAGs) and other components of the endothelial glycocalyx decreased dramatically after superfusion of the mesentery with the chemoattractant $N$-formylmethionyl-leucyl-phenylalanine and during reperfusion after 60 -min ischemia. These reductions were significantly attenuated by superfusion with pertussis toxin (PTX), suggesting that shedding of glycocalyx was mediated by $\mathrm{G}$ proteins. Adhesion of microspheres linked with antibody for syndecan-1, a major proteoglycan to which GAGs are bound, revealed increased labeling as GAGs were lost and permitted greater numbers of spheres to adhere to the protein core, which was not shed. Induction of ischemia by occluding proximal microvessels for 60 min resulted in a $40 \%$ increase in galactosaminoglycans and a $15 \%$ increase in glucosaminoglycans on the endothelium, which was not inhibited by PTX. Reperfusion of vessels led to a rapid loss of GAGs that was inhibited by pretreatment with PTX, with $40 \%$ of galactosaminoglycans and $25 \%$ of glucosaminoglycans accumulated being removed by $\mathrm{G}$ protein-mediated shedding and the remainder freely convected away by fluid shear. We conclude that the composition of the glycocalyx results from a balance of the rate of biosynthesis of GAGs by the endothelial cell and their shedding, which may be mediated by intracellular and/or membrane-bound proteases or lyases released or activated by $\mathrm{G}$ protein signaling.
\end{abstract}

endothelium; venules

IT IS WELL RECOGNIZED THAT the glycocalyx of the endothelial cell (EC) serves as a barrier to transvascular exchange of macromolecules and blood cell adhesion to the endothelium and may affect the resistance to blood flow within the microcirculation proper. Within the microvasculature, observations by electron microscopy using cationic markers reveal that the glycocalyx is comprised of a highly sulfated layer of glycosaminoglycans (GAGs) bound to a variety of proteins (proteoglycans) that is on the order of $0.1 \mu \mathrm{m}$ in thickness (2). In situ studies of the exclusion of blood cells from the plasma membrane of microvascular ECs have shown that the glycocalyx may extend much farther into the capillary lumen, to on the order of $500 \mathrm{~nm}$ (36). It has been suggested that the glycocalyx is part of a heterogeneous endothelial surface layer that extends to almost $1 \mu \mathrm{m}$ from the EC membrane (24). In vivo microvascular studies of enzymatic degradation of specific GAGs have revealed dramatic increases in capillary hematocrit (7), increased capillary permeability (1), enhanced leukocyte [white blood cell (WBC)] adhesion (20), and diminished resistance to blood flow within the microcirculation (25). It has also been

Address for reprint requests and other correspondence: H. H. Lipowsky, Dept. of Bioengineering, Penn State Univ., 233 Hallowell Bldg, Univ. Park, PA 16802 (E-mail: hhlbio@engr.psu.edu). shown that the composition of the glycocalyx changes in microvessels during angiogenesis (11) and with onset of atherosclerosis in macrovessels (6).

Heparan sulfate (HS), a glucosaminoglycan and the most common GAG on the EC glycocalyx, is associated with $\sim 50$ $90 \%$ of endothelial proteoglycans (14) and is typically present in a ratio of 4:1 with the second most common GAG, the galactosaminoglycan chondroitin sulfate (CS) (26). The GAGs dermatan and keratin sulfate may also be present on the EC to a lesser extent than CS and HS (34). The principal proteins on the EC surface that bind HS and CS to form the proteoglycans are the transmembrane syndecans and the membrane-bound glypicans (24). The most prevalent proteoglycan, syndecan-1, is one of four members of this family of proteoglycans and is a type I integral membrane protein composed of an intracellular domain, a transmembrane domain, and an extracellular core (29). Syndecan-1 typically has one to three HS and one to three CS GAG molecules attached to its core protein. The number of GAGs attached to the core protein is variable and may depend on the physiological state and location of the tissue. Studies of syndecan-1 knockout mice have suggested that its absence leads to enhanced WBC-EC adhesion, although the extent to which the WBCs or the ECs contribute to this response remains to be determined (10).

It has also been suggested that the GAG hyaluronan (HA), which is not bound to a proteoglycan core, may play a role in permeation of the glycocalyx by macromolecules (12) and contribute to the structural integrity of the GAG protein matrix (37). However, the proportion of HA relative to HS and CS remains to be determined.

There is sufficient in vitro evidence to suggest that components of the glycocalyx are shed in response to signal transduction events common to inflammation and ischemia-reperfusion. Syndecan-1 has been shown to be shed after treatment by thrombin and epidermal growth factors, and its shedding can be mediated by G protein-coupled receptors (GPCRs), protein tyrosine kinase receptors, and a tissue inhibitor of the metalloproteinase-3-sensitive pathway $(9,34)$. HS is shed after the treatment of cultured ECs with complement (13) and endotoxin (5). Prior studies suggested that stimulation of the GPCRs with $N$-formylmethionyl-leucyl-phenylalanine (fMLP) results in enhanced WBC adhesion in postcapillary venules in mesentery (rat) because of shedding of HS proteoglycans that in turn exposes greater amounts of ICAM-1 to facilitate adhesion of the CD11a,b/CD18 receptor on the leukocyte (20). Activation of human monocytic THP-1 cells with fibronectin and IFN- $\gamma$ results in enhanced binding of $\mathrm{Fc}$ receptors (CD32) to antibody because of shedding of CD43 (leukosialin) from its glycocalyx (28).

Within this framework, the present study was undertaken to examine the shedding of specific components of the glycocalyx

\footnotetext{
The costs of publication of this article were defrayed in part by the payment of page charges. The article must therefore be hereby marked "advertisement" in accordance with 18 U.S.C. Section 1734 solely to indicate this fact.
} 
of postcapillary venular endothelium in intestinal mesentery (rat) during a well-defined model of inflammation and ischemia-reperfusion and thus test the hypothesis that shedding of the glycocalyx facilitates greater WBC-EC adhesion during these events. To probe for alterations in the composition of the glycocalyx, two approaches were followed: 1) alterations in glucosaminoglycan and galactosaminoglycan moieties were delineated on the surface of the glycocalyx with lectin- or antibody-coated fluorescent microspheres $(0.1 \mu \mathrm{m}$ in diameter), and 2) fluorescently labeled lectins were used to probe deeper into the endothelial surface layer. The adhesion of these markers to the walls of postcapillary venules was quantified under resting (control) conditions and during a stimulated inflammatory response elicited by the topical application of fMLP, which resulted in a decrease in these labels for glucosaminoglycans and galactosaminoglycans. To elucidate the effects of hydrodynamic shear on the composition of the glycocalyx, the binding of lectin-labeled microspheres and lectins alone was studied during normoxic ischemia induced by a blunted microprobe to stop flow in selected venules. It was found that the removal of shear resulted in an accumulation of lectins on the EC in a pertussis toxin (PTX)-insensitive mechanism. Reperfusion of the vessels led to the shedding of the glycocalyx in a $\mathrm{G}$ protein-mediated mechanotransduction mechanism, as assessed by its inhibition with PTX.

\section{MATERIALS AND METHODS}

\section{Animal Preparation}

All studies were conducted in conformance with the "Guiding Principles for Research Involving Animals and Human Beings" of the American Physiological Society. Male Sprague-Dawley rats weighing $300-450 \mathrm{~g}$ were anesthetized with inactin $(125 \mathrm{mg} / \mathrm{kg}$ ip), tracheostomized, and allowed to breathe spontaneously. The right internal jugular vein was cannulated with polyethylene (PE-50) tubing to enable administration of supplemental doses of anesthetic as required to maintain a surgical plane of anesthesia. The left carotid artery was cannulated with PE-90 tubing and connected to a strain gauge-type pressure transducer to monitor arterial blood pressure. To facilitate systemic infusion of fluorescently labeled microspheres (FLMs), the right femoral vein was cannulated with PE-10 tubing and connected to an infusion syringe pump (Harvard Apparatus, Holliston, MA). Systemic hematocrit $\left(\mathrm{H}_{\mathrm{sys}}\right)$, WBC count, and circulating FLM concentration $\left([\mathrm{FLM}]_{\text {circ }}\right)$ were measured by withdrawing $0.25 \mathrm{ml}$ of blood through the carotid artery catheter. Leukocyte counts were obtained with a Coulter counter (model ZM; Beckman-Coulter, Miami, FL). For all animals studied, the WBC count averaged 5,556.5 \pm 1,727.8 cells $/ \mu \mathrm{l}$ and $\mathrm{H}_{\text {sys }}$ averaged $49.2 \pm 2.1 \%$. FLM counts were obtained by using a hemocytometer while viewing under a fluorescence microscope (Nikon Microphot) with a Zeiss water immersion $\times 40$, 0.75-numerical aperture (NA) objective.

\section{Intravital Microscopy and Experimental Protocols}

The intestinal mesentery was exteriorized through a midline abdominal incision and placed on a glass pedestal to facilitate viewing under either brightfield microscopy by transillumination or incident illumination with a Ploeme-type fluorescence illuminator with fluorescein excitation and emission filters. The tissue was suffused with HEPES-buffered Ringer solution $(\mathrm{pH}=7.4)$ at a temperature of $37.0^{\circ} \mathrm{C}$. Solutions of fMLP $\left(10^{-7} \mathrm{M}\right.$; Sigma, St. Louis, MO) and PTX (100 ng/ml; Sigma) were prepared in HEPES-buffered Ringer solution $(\mathrm{pH}=7.4)$ for irrigation of the tissue. The protocol used for these experiments was as follows. A bolus of labeled FLMs was infused at
$2 \times 10^{12}$ spheres $\cdot \mathrm{ml}^{-1} \cdot \mathrm{kg}^{-1}$ to obtain a $[\text { FLM }]_{\text {circ }}$ of $10^{7} / \mathrm{ml}$ and maintained at that level by intravenous infusion of $2 \times 10^{9}$ spheres $\mathrm{kg}^{-1} \cdot \mathrm{min}^{-1}$ via the femoral vein. To simulate the inflammatory process, after a control period tissues were superfused with either fMLP $\left(10^{-7} \mathrm{M}\right)$-Ringer or normal Ringer solution for the duration of the experiment. One-half of the tissues superfused with fMLP-Ringer received pretreatment with PTX $(100 \mathrm{ng} / \mathrm{ml})$ in Ringer solution for 30 min. To study the effects of prolonged shear rate reduction, a condition of normoxic ischemia was induced by occluding a proximal arteriole with a blunted glass micropipette for a period of $60 \mathrm{~min}$. Hypoxia was prevented by constantly superfusing the tissue with Ringer solution equilibrated to room air. To study the role of $G$ proteins signaling during ischemia and reperfusion, the mesenteric tissue was superfused with Ringer solution plus PTX (100 ng/ml). The result of this treatment was compared with behavior in nonoccluded venules in the same tissue.

Mesenteric postcapillary venules ranging in width from 25 to 50 $\mu \mathrm{m}$ were viewed with a Zeiss water immersion $\times 40,0.75-\mathrm{NA}$ objective for measurement of red blood cell velocity $\left(V_{\mathrm{RBC}}\right)$ and counting microspheres adhered to the EC. The image was projected onto a low-light level silicon-intensified target camera (model 66; Dage-MTI, Michigan City, IN) for an effective width of the video field of $100 \mu \mathrm{m}$. By focusing above and below the microvessel diametral plane during video recording, all of the intensely fluorescent microspheres could be counted during offline analysis.

Center line $V_{\mathrm{RBC}}$ was measured in arterioles and venules with the two-slit photometric technique (self-tracking correlator; IPM, San Diego, CA) as described previously (20). The mean velocity of blood $\left(V_{\text {mean }}\right)$ was calculated from the relationship $V_{\text {mean }}=V_{\mathrm{RBC}} / 1.6$ (19). Microvessel diameter was measured by the video image shearing technique with an image shearing monitor (IPM).

\section{Confocal Microscopy}

The concentration of fluorescently labeled lectins along the EC was measured with confocal microscopy on fixed mesenteric tissues for both the inflammation and ischemia-reperfusion models. Tissue samples were fixed by rapid superfusion with $2.5 \%$ glutaraldehyde in Ringer solution. Mesenteric tissues were excised and viewed with an Olympus FV300 confocal microscope and a $\times 60$ oil immersion, 1.4-NA objective.

\section{Fluorescent Microsphere Preparation and Lectin Labeling}

Fluorescent (yellow-green) carboxylate-modified polystyrene microspheres, $0.1 \mu \mathrm{m}$ in diameter (Fluospheres; Molecular Probes, Eugene, OR), were labeled by covalent linkage of one of the following molecules: 1) antiglucosaminoglycan Lycopersicon esculentum lectin (LEA; Sigma), 2) antigalactosaminoglycan Bandeiraea simplicifolia lectin (BS1; Sigma), 3) anti-HS MAb F58-10E4 (Associates of Cape Cod, Falmouth, MA), 4) anti-CS MAb CS56 (Sigma), 5) anti-syndecan-1 MAb H174 (Santa Cruz Biotechnology, Santa Cruz, CA), and 6) an IgM isotype MAb (Zymed, San Francisco, CA) to rule out nonspecific binding. To confirm the specificity of FLMs coated with LEA and BS1 for carbohydrates, labeled FLMs were incubated (for $60 \mathrm{~min}$ ) in the hapten molecules chitotriose (1 mM; Sigma) or raffinose (5 mM; ICN Pharmaceuticals), respectively, to inhibit their binding to GAGs on the EC. All FLMs were labeled with the carbodiimide reaction to attach the desired protein to the carboxylatecoated FLMs as previously detailed (20). Lectins were also directly labeled with FITC by incubation in FITC $(0.05 \mathrm{mg} / \mathrm{mg}$ lectin) in carbonate/bicarbonate buffer $(\mathrm{pH}=9)$ and then dialyzed against PBS to remove excess FITC.

\section{Measurements}

WBC and FLM adhesion. The numbers of WBCs and FLMs firmly adhered were measured by frame-by-frame analysis of video record- 
ings and quantified as the number adhering per $100 \mu \mathrm{m}$ of venule length. Adhered WBC and FLMs were judged to be firmly adhered if they remained stationary for at least $5 \mathrm{~s}$. All FLMs and WBC were counted by frame-by-frame playback of video recordings obtained while focusing the microscope objective above and below the diametral plane.

Lectin concentration. The concentration of fluorescently labeled lectin along the vascular endothelium was determined by confocal microscopy. The fluorescence intensity at the vessel midline $\left(\mathrm{I}_{\mathbf{M}}\right.$, proportional to residual concentration of circulating lectins) and the background tissue intensity $10 \mu \mathrm{m}$ outside the vessel wall $\left(\mathrm{I}_{\mathrm{B}}\right.$, due to tissue autofluorescence) were subtracted from the intensity at the vessel wall $\left(\mathrm{I}_{\mathrm{W}}\right)$. The corrected wall intensity was converted to a concentration of fluoresceinated lectins with calibration curves obtained for known standards in $50-\mu \mathrm{m}$ path length cuvettes placed under the confocal microscope. Statistical analyses of trends in the data were performed with SigmaStat (SPSS) and used either Student's $t$-test for paired tests or the Holm-Sidak method for ANOVA of multiple comparisons. Values are expressed as means \pm SD unless otherwise noted.

\section{RESULTS}

\section{Glycosaminoglycan Shedding in Response to fMLP}

Presented in Fig. 1 is the time course of FLM adhesion before and after superfusion of the tissue with fMLP for 1 ) microspheres labeled with $\mathrm{BS} 1$, to detect galactosaminoglycans, and 2) microspheres labeled with LEA, to detect glucosaminoglycans. The time scale was adjusted to zero at the initiation of fMLP superfusion. Without any treatments (controls), as sphere binding reached steady-state values the number of BS1-labeled spheres averaged $10.6 \pm 1.78$ spheres/100 $\mu \mathrm{m}$ of venule length (Fig. 1A) and LEA-labeled spheres averaged $17.9 \pm 4.47$ spheres/100 $\mu \mathrm{m}$. Preincubation of the FLMs in raffinose or chitotriose, to inhibit binding to galactosaminoglycans and glucosaminoglycans, respectively, revealed an insignificant level of FLM adhesion throughout the time course of the experiment. Raffinose blocked $89 \%$ of control adhesion and revealed maximum adhesion values of $1.27 \pm 2.13$ [under control conditions, time $(t)<0$ ] and $1.21 \pm 2.6$ (with fMLP superfusion, $t>0$ ) spheres/100 $\mu \mathrm{m}$ (Fig. 1A). Chitotriose blocked $95 \%$ of control adhesion and revealed maximum adhesion values of $0.96 \pm 1.10$ (control conditions, $t<0$ ) and $2.72 \pm 1.04$ (with fMLP superfusion) spheres/100 $\mu \mathrm{m}$ (Fig. 1B).

After superfusion of the tissue with fMLP, adhesion of the BS1-labeled FLMs fell significantly $(P<0.006)$ below levels in control vessels (Fig. 1A). Pretreatment of vessels with the superfusion of PTX (100 ng/ml in Ringer solution) prevented significant loss of BS1 FLM adhesion and resulted in adhesion values that were not significantly different $(P=0.378)$ from control values and were significantly greater than those in fMLP-treated microvessels $(P<0.026)$. These trends represent a significant loss in galactosaminoglycans during fMLP superfusion.

Similar experiments were performed with LEA-coated FLMs (Fig. 1B). Control vessels (untreated) maintained an adhesion level of $17.9 \pm 4.47 \mathrm{FLM} / 100 \mu \mathrm{m}$, compared with fMLP-treated vessels, which experienced a rapid and significant decrease to $8.9 \pm 2.27 \mathrm{FLM} / 100 \mu \mathrm{m}(P<0.045)$. Pretreatment with PTX superfusion significantly inhibited the fMLP-induced decrease in LEA FLM adhesion, resulting in adhesion levels that were not significantly different from con-
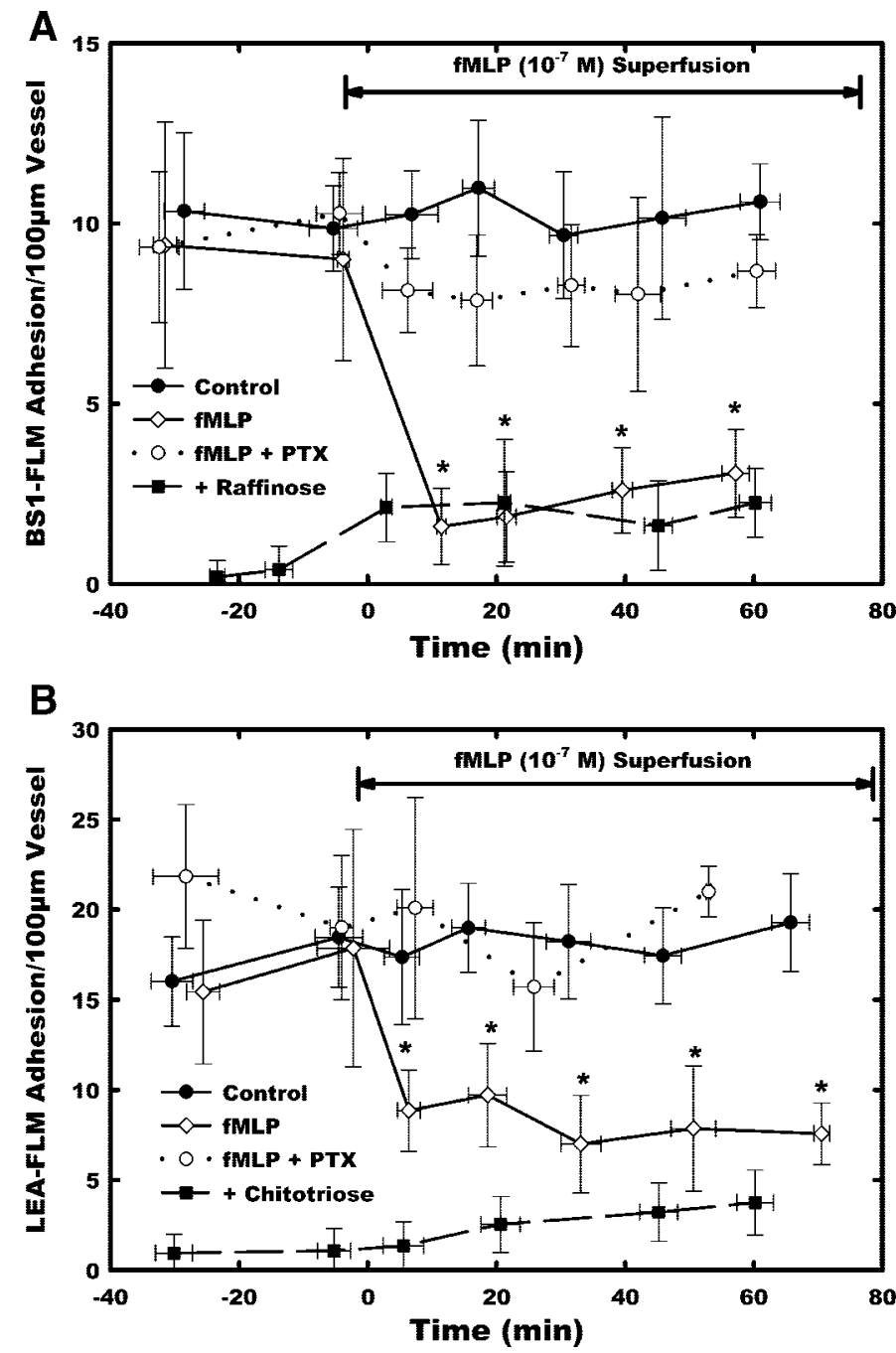

Fig. 1. Adhesion of lectin-coated fluorescently labeled microspheres (FLMs), linked to Bandeiraea simplicifolia lectin (BS1) for galactosaminoglycans (A) and Lycopersicon esculentum lectin (LEA) for glucosaminoglycans (B). Given are the number of FLMs adhered per $100-\mu \mathrm{m}$ venule length under control conditions and in response to superfusion of the mesentery with $10^{-7} \mathrm{M}$ $N$-formylmethionyl-leucyl-phenylalanine [fMLP; applied at time $(t)=0$ ], which resulted in a significant decrease from control, indicative of shedding of glycosaminoglycan (GAG) components ( $n=17$ venules each). Also shown is the response to fMLP after 30 min of superfusion with pertussis toxin (PTX, $100 \mathrm{ng} / \mathrm{ml} ; n=10$ ), which inhibited GAG shedding and was not significantly different from control $(n=12)$. To validate specificity of the lectins, FLMs were preincubated in vitro $(1 \mathrm{~h})$ in the haptens chitotriose $(n=8)$ or raffinose $(n=10)$, which resulted in insignificant adhesion. Values are means \pm SD. *Significantly different from control $(P<0.05)$.

trol $(P=0.232)$. Thus glucosaminoglycans were also significantly reduced during fMLP superfusion.

Adhesion of FLMs coated with the anti-CS MAb CS-56 and the anti-HS MAb F58-10E4 were also examined during control conditions and fMLP superfusion. Infusion of spheres with a nonbinding isotype (IgM FLMs) resulted in mean values under control conditions (3.38 $\pm 2.62 / 100 \mu \mathrm{m})$ and after superfusion with fMLP $(3.54 \pm 1.65 / 100 \mu \mathrm{m})$, which were not significantly increased from control values $(P=0.327)$. During the initial control period, adhesion of the CS56 FLMs averaged $2.71 \pm 1.99 \mathrm{FLM} / 100 \mu \mathrm{m}$ and did not reach adhesion levels significantly greater $(P=0.534)$ than the IgM FLMs 


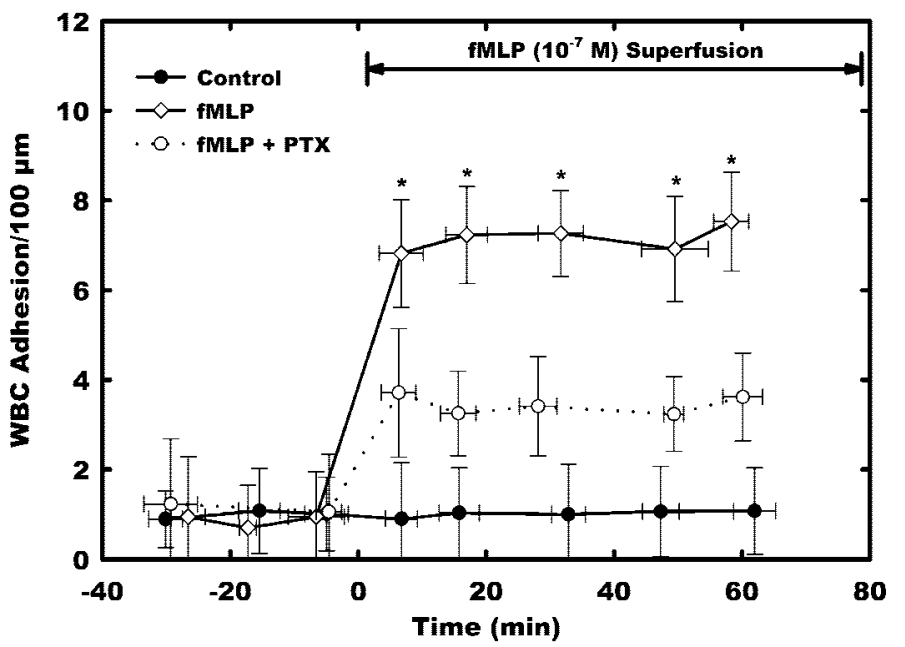

Fig. 2. Number of leukocytes [white blood cells (WBCs)] adhered per $100-\mu \mathrm{m}$ venule length during BS1 FLM and LEA FLM experiments shown in Fig. 1 $(n=34)$. The increased number of adhering WBCs in response to superfusion of the mesentery with fMLP is typical for rat mesentery. Superfusion of the mesentery with PTX (100 ng/ml in Ringer solution) for $30 \mathrm{~min}$ before fMLP significantly inhibited WBC-endothelial cell (EC) adhesion $[n=20$; not different from control (Ringer only, $n=25$ )]. Values are means \pm SD. * Significantly different from control $(P<0.05)$.

during control conditions (data not shown). In contrast to the lectin FLMs, during fMLP superfusion adhesion of the CS56 FLMs increased only slightly to $5.86 \pm 6.31 \mathrm{FLM} / 100 \mu \mathrm{m}$ and was not significantly greater than either the IgM FLM $(P=$ $0.536)$ or the CS56 FLM control values $(P=0.078)$. The lack of consistency between the MAb to CS and lectin labeled spheres to galactosaminoglycans was attributed to a combination of steric hindrance of the large IgM isotype and poor avidity for the intact CS.

In contrast, the adhesive response of FLMs labeled with $\mathrm{MAb}$ for HS followed the trends delineated by the lectinlabeled FLMs. Anti-HS F58-10E4 FLMs averaged $11.4 \pm 7.4$ FLM/100 $\mu \mathrm{m}$ during the initial control period, which was significantly greater than the IgM FLM adhesion during control conditions $(P<0.001$; data not shown). Adhesion of the F58-10E4 FLMs fell significantly to $7.72 \pm 4.4 \mathrm{FLM} / 100 \mu \mathrm{m}$ during $30 \mathrm{~min}$ of fMLP superfusion $(P<0.046)$.

\section{Leukocyte-Endothelium Adhesion}

Levels of WBC adhesion during the BS1 FLM and LEA FLM experiments are shown in Fig. 2. During the FLM infusion experiments the untreated vessels had a control level of WBC adhesion of $1.01 \pm 0.07 \mathrm{WBC} / 100 \mu \mathrm{m}$. Superfusion of fMLP caused a significant increase $(P<0.002)$ in WBC adhesion to attain a value of $7.15 \pm 0.29 \mathrm{WBC} / 100 \mu \mathrm{m}$. Treatment of vessels with PTX before fMLP superfusion significantly attenuated WBC adhesion to $3.45 \pm 0.22 \mathrm{WBC} /$ $100 \mu \mathrm{m}$, which is significantly less than the fMLP treatment alone $(P<0.017)$ and not significantly different from control values $(P=0.146)$.

\section{Syndecan-1 Exposure During fMLP Stimulation}

Levels of the proteoglycan syndecan-1 during fMLP stimulation were examined by using FLMs labeled with the monoclonal antibody H174, which is specific for the syndecan-1 core protein, as shown in Fig. 3. Both control and fMLP treatments showed a steady rise in FLM adhesion over the first $30 \mathrm{~min}(t=-20$ to $10 \mathrm{~min})$. This trend was attributed to the asymptotic buildup to a steady state as evidenced by the control FLM data. In response to fMLP, H174 FLM adhesion increased twofold above control $(P<0.001)$. Linear regression of the number of adhered FLMs vs. time for control vessels yielded a small yet significant slope of 0.0147 FLM•100 $\mu \mathrm{m}^{-1} \cdot \mathrm{min}^{-1}(P<0.004)$. There was no significant difference $(P=0.244)$ between the control and fMLP-treated vessels before fMLP superfusion. After 25-min fMLP superfusion, however, the adhesion of H174 FLM increased significantly $(P<0.018)$ from control values of $24.98 \pm 6.48$ to $38.23 \pm$ 7.66 FLM/100 $\mu \mathrm{m}$. Thus it appears that the core protein (syndecan-1) remains intact during fMLP stimulation and greater amounts are exposed for binding with the MAb FLMs as GAGs are shed.

\section{Binding of Fluorescently Labeled Lectins}

To explore the steric hindrance of $0.1-\mu \mathrm{m}$-diameter microspheres, studies were conducted to assay the binding of fluoresceinated lectins alone, estimated to have a hydrodynamic diameter of $\sim 0.01 \mu \mathrm{m}$. Animals were given a bolus of either BS1 or LEA labeled with FITC at a concentration of $2.5 \mathrm{mg} / \mathrm{kg}$ to obtain a homogeneous coating of the vascular endothelium, as described in MATERIALS AND METHODS. Illustrated in Fig. $4 A$ is a representative image of the accumulation of BS1-FITC in the glycocalyx of a postcapillary venule $15 \mathrm{~min}$ after infusion. The corresponding brightfield image is shown in Fig. $4 B$. The enlarged image of a section of this venule shown in Fig. $4 C$ illustrates the approach to quantitating the intensity of this fluorophore. The dashed line delineates the ensemble of image pixels that comprise the intensity distribution shown in Fig. $4 D$. The peak wall intensity was corrected for background fluorescence in the tissue and vessel lumen and converted to a concentration of lectin with standards of prescribed concentration.

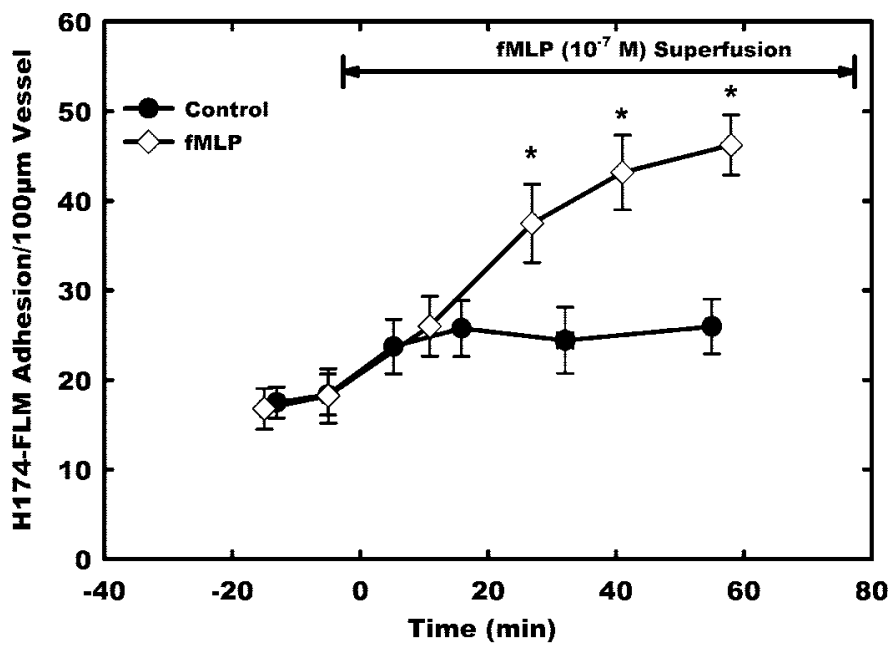

Fig. 3. Adhesion of FLMs coated with MAb for syndecan-1 (H174 FLMs) per $100-\mu \mathrm{m}$ venule length during control $(n=8)$ and superfusion with $\operatorname{fMLP}(n=$ 11). Adhesion of H174 FLMs increased significantly during fMLP treatment because of greater access to the core protein as steric hindrance was reduced with GAG shedding. Values are means \pm SD. *Significantly different from control $(P<0.05)$. 
Fig. 4. Representative postcapillary venule stained with BS1-FITC under confocal fluorescence $(A)$ and transmitted brightfield microscopy $(B)$. Rats were infused with BS1FITC $(2.5 \mathrm{mg} / \mathrm{kg})$, and the mesentery was fixed by topical application of $2.5 \%$ glutaraldehyde in Ringer solution at specific times after initiation of superfusion with $10^{-7} \mathrm{M}$ fMLP. In $D$ the image of the venule was enlarged and the intensity of the fluorophore was measured along the radial line, as shown in $C$. The peak fluorescence intensity of the wall was corrected for background fluorescence by subtracting the fluorescence intensity within the vessel lumen (arising from free dye) and the surrounding tissue (arising from tissue autofluorescence). WBCs were also labeled by the lectins and are visible.
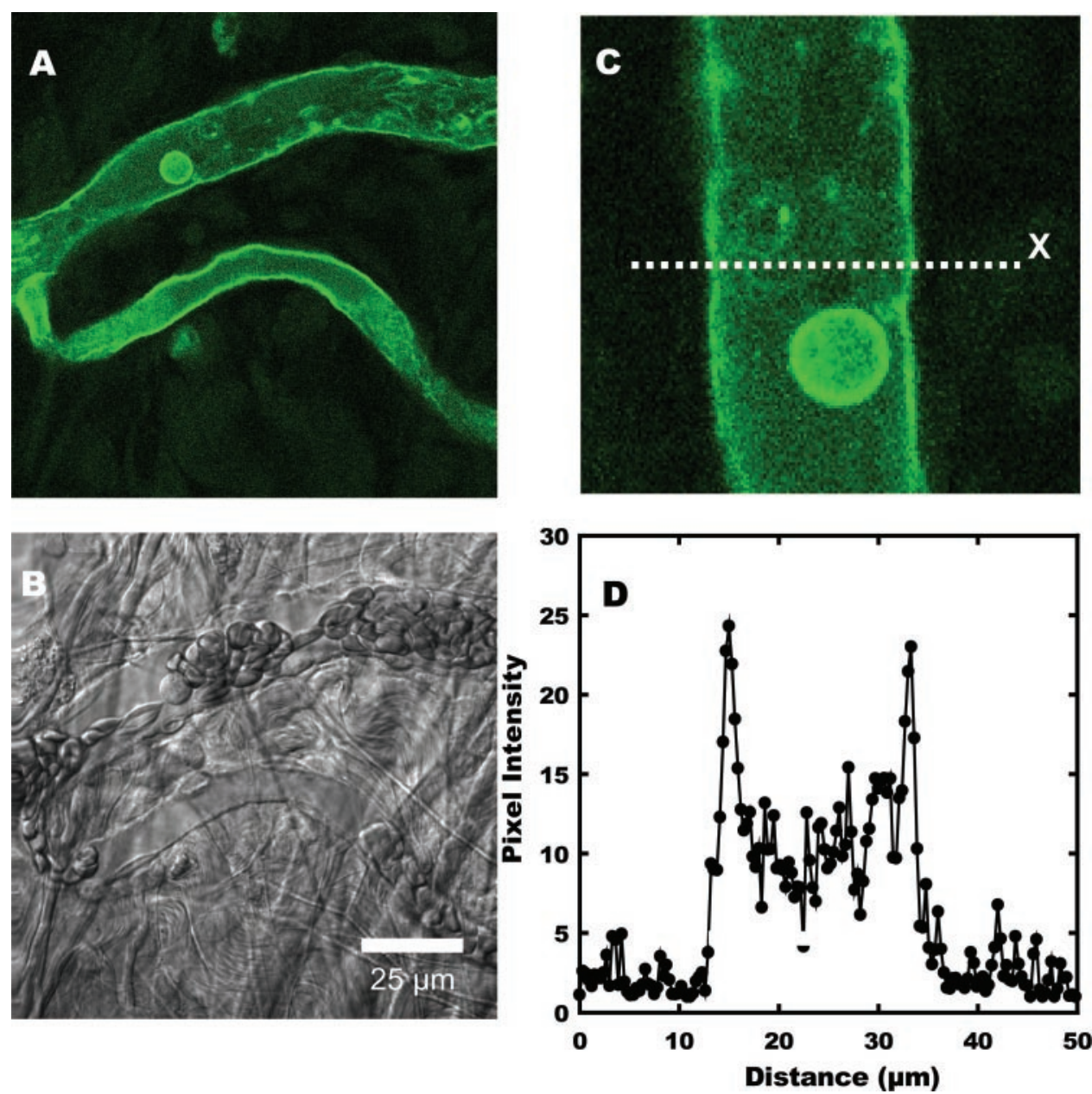

The peak wall concentration of BS1-FITC during control, fMLP superfusion, and PTX followed by fMLP treatment is shown in Fig. 5A. The effects of fMLP were observed within 5 min, when the concentration of BS1-FITC had decreased significantly by almost $50 \%(P<0.001)$. The concentration of BS1-FITC remained significantly lower than control values at $15(P<0.001), 30(P<0.001)$, and $45(P<0.003)$ min after fMLP superfusion. The superfusion of PTX (in Ringer solution) before fMLP significantly inhibited the previously seen fMLP-induced decreases in BS1-FITC. PTX-treated tissues yielded BS1-FITC concentrations along the EC that were not significantly different from those in untreated (control) vessels at $5(P=0.308), 15(P=0.099), 30(P=0.376)$, and $45(P=$ $0.945) \mathrm{min}$. There was a significant difference in the concentration of BS1-FITC on the vessel wall between the fMLP- and the fMLP+PTX-treated vessels. The PTX had a noticeable and significant effect in inhibiting the fMLP-induced loss of BS1FITC on the EC at $5(P<0.001), 15(P<0.001), 30(P<$ $0.001)$, and $45(P=0.003) \mathrm{min}$. The results of the BS1-FITC experiments were in accord with the BS1-FLM experiments and suggest a loss in galactosaminoglycans after fMLP superfusion.

There was no significant decrease in LEA-FITC concentration along the vessel wall at $15 \min (P=0.343$; Fig. $5 B)$. However, at $30(P=0.04)$ and 45 min after fMLP treatment the concentration of LEA-FITC decreased significantly $(P=$
0.021). The results of the LEA-FITC confocal experiments agree with the LEA-FLM experiments and suggest that there is a loss of glucosaminoglycans during fMLP superfusion. In the FLM experiments the time to achieve a significant decline in concentration was not significantly different for either glucosaminoglycans or galactosaminoglycans. With lectin-FITC there was a longer time to attain a significant drop in glucosaminoglycan concentration on the wall compared with galactosaminoglycan concentration. Inhibition of LEA-FITC binding with PTX was not attempted because of the large standard deviations and relatively small reduction of total glucosaminoglycan shed in response to fMLP.

\section{GAG Behavior During Ischemia}

The effects of shear on GAG behavior in venules were studied by occluding vessels for $60 \mathrm{~min}$ and comparing the adhesion of lectin FLMs and lectin-FITC to that of unoccluded vessels within the same tissue. The adhesion of BS1 FLMs and LEA FLMs during flow occlusion and during reperfusion is shown in Fig. 6, $A$ and $B$, respectively. For each treatment, the number of FLMs adhered to the venular wall was averaged for four to six control (patent) and four to six ischemic (occluded) venules. FLM adhesion was normalized for each tissue by taking the ratio of these two averages. Adhesion of the BS1 FLMs to ECs increased significantly by almost $25 \%(P<$ 

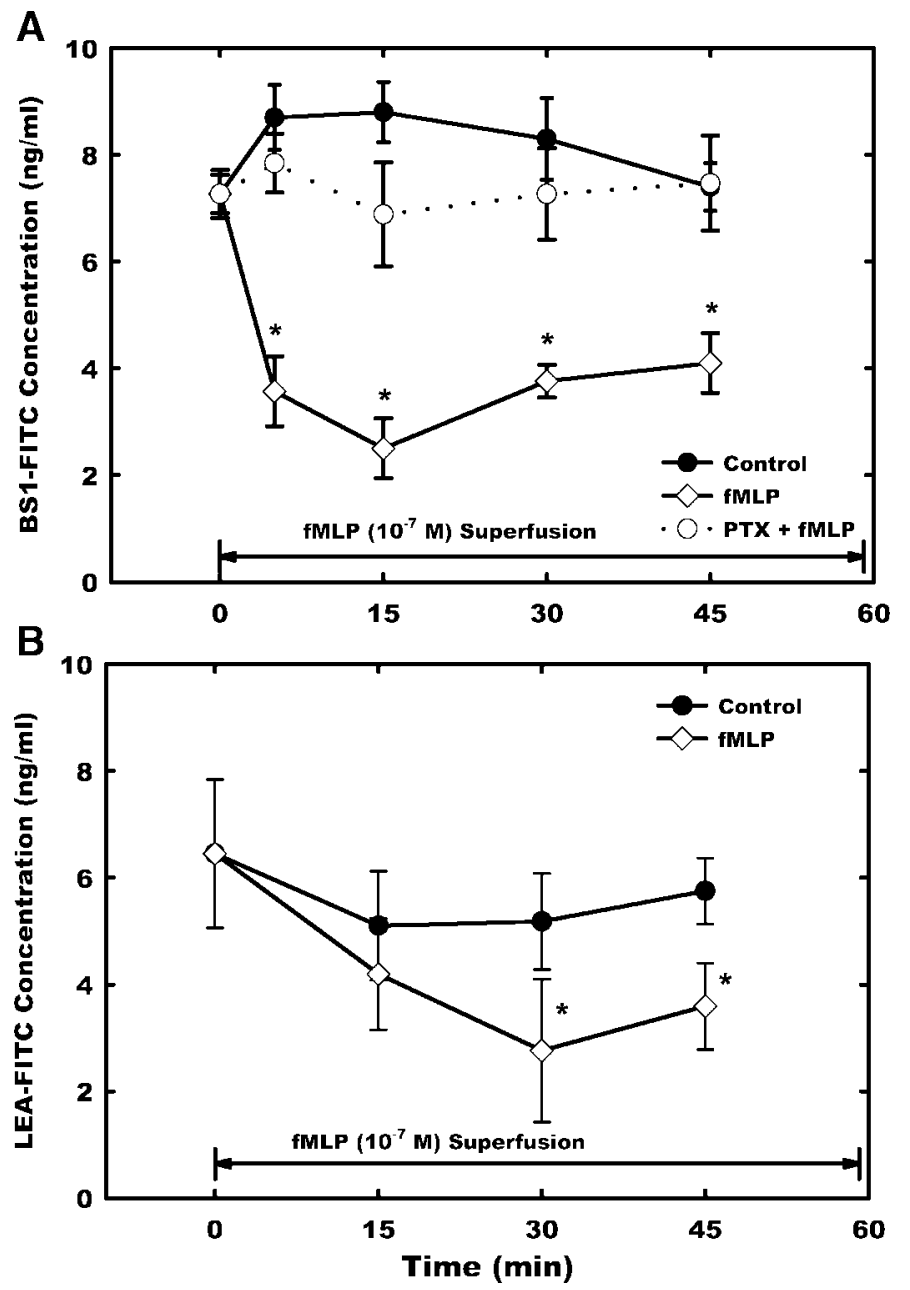

Fig. 5. Time course of BS1-FITC $(A)$ and LEA-FITC $(B)$ binding to the EC after superfusion with fMLP, as determined by confocal microscopy. Lectins were infused at $t=-10 \mathrm{~min}$, and fMLP application began at $t=0$. The intensity of the fluorescence was converted to a concentration with a set of known standards. The substantially smaller lectin is believed to infiltrate the entire thickness of the glycocalyx (in contrast to the FLMs that label only the surface), as suggested by the longer time to reach a minimum as GAGs are shed. The 3-fold increase in time to shed glucosaminoglycan, compared with galactosaminoglycan, is attributed to its greater concentration in the glycocalyx. Values are means \pm SD for 30 venules in each treatment. *Significantly different from control $(P<0.05)$

0.001) during the 60 min of flow occlusion. Pretreatment with PTX had no effect on the buildup of either BS1 FLMs or LEA FLMs during occlusion. During reperfusion the occluded vessels incurred a $15 \%$ decrease in BS1 FLM adhesion by $15 \mathrm{~min}$ of reperfusion. The decrease in BS1 FLM adhesion during reperfusion was inhibited by PTX, suggesting that the mechanism of loss was $G$ protein related. Adhesion of the LEA FLMs to the EC increased significantly $(P<0.001)$ by almost $40 \%$ during the 60 min of flow occlusion. The increase was similar for occluded vessels that had been pretreated with PTX, suggesting that $G$ proteins had no role in the buildup during occlusion and that shear was the main factor. During reperfusion the occluded vessels exhibited a significant $(P<0.001)$ $20 \%$ drop in LEA FLM adhesion by 15 min of reperfusion. This drop in LEA FLM was inhibited by PTX, suggesting a G protein-dependent mechanism.
The absence of significant changes in $V_{\mathrm{RBC}}$ (Fig. 6, $C$ and $D$ ) during the BS1 FLM and LEA FLM flow occlusion experiments suggests that changes in flow or wall shear rate between control and occluded/reperfused vessels did not contribute to the results. Estimates of wall shear rates averaged $442.3 \pm$ $76.46 \mathrm{~s}^{-1}$ and were invariant throughout the protocol.

The concentrations of BS1-FITC and LEA-FITC were measured along the vascular endothelium in vessels that had been occluded (no flow) for $60 \mathrm{~min}$ and were then reperfused (Fig. 7 ). The data were normalized with respect to average values in nonoccluded venules in the same tissue preparation. After 60 min of flow occlusion and before flow was reestablished, the occluded vessels showed a significant increase $(P<0.001)$ in BS1-FITC concentration compared with the nonoccluded (control) vessels (Fig. 7A). After $5 \mathrm{~min}$ of reestablished flow the concentration of BS1-FITC decreased significantly $(P<$ 0.001) compared with the buildup during occlusion, but this value was not significantly different $(P=0.096)$ from control vessels. At $10 \mathrm{~min}$ after flow was reestablished the BS1-FITC concentration in the occluded vessels had decreased further and was significantly lower $(P<0.001)$ than that in the control vessels. At 15 and $30 \mathrm{~min}$ after reestablished flow the BS1FITC concentration approached control values $(P=0.248$ and $P=0.032$, respectively). The superfusion of PTX had no significant effect on the initial buildup of BS1-FITC after 60 min of flow occlusion compared with the occlusion-only vessels $(P=0.106)$. However, PTX was able to significantly inhibit any decreases in BS1-FITC concentration observed after reperfusion. There was no significant difference between PTX-treated vessels and control vessels during reperfusion at $5,10,15$, and $30 \mathrm{~min}(P=0.48,0.311,0.896$, and 0.78 , respectively).

After $60 \mathrm{~min}$ of flow occlusion and before flow was reestablished, the occluded vessels showed a significant increase $(P<0.001)$ of $\sim 20 \%$ in LEA-FITC concentration compared with the nonoccluded (control) vessels (Fig. 7B). After $5 \mathrm{~min}$ of reestablished flow the concentration of LEA-FITC decreased significantly $(P<0.001)$ compared with the buildup during occlusion, but this value was not significantly different $(P=0.35)$ from that in control vessels. At 15 min after reestablished flow the LEA-FITC concentration in the occluded vessels had decreased further and was significantly lower $(P<0.001)$ than the control vessels. At 30 min after reperfusion of flow began the LEA-FITC concentration approached control values $(P=0.08)$. Superfusion with PTX had no significant effect on the initial buildup of LEA-FITC after $60 \mathrm{~min}$ of flow occlusion compared with the occlusion-only vessels $(P=0.82)$. However, PTX significantly inhibited decreases in LEA-FITC concentration after reperfusion. A significant difference between PTX-treated and occluded vessels occurred during reperfusion at 15,25 , and $30 \mathrm{~min}(P<$ $0.001, P<0.001$, and $P=0.005$, respectively). As in the case of the fMLP protocols, shedding of glucosaminoglycans (Fig. 7B) appeared to lag behind that of galactosaminoglycans (Fig. 7A).

\section{DISCUSSION}

The present studies support the hypothesis that the composition of the glycocalyx results from a balance of the rate of biosynthesis of GAGs by the EC and their shedding, which may be mediated by intracellular and/or membrane-bound 
Fig. 6. Time course of lectin-labeled FLMs during ischemia (60-min proximal occlusion by a microprobe) followed by reperfusion. Adhesion of microspheres coated with lectin BS1 $(A)$ for galactosaminoglycans and lectin LEA $(B)$ for glucosaminoglycans, per $100-\mu \mathrm{m}$ venule length, was normalized with respect to FLM adhesion counts in nonoccluded venules in the same tissue. Vessels were occluded from $t=-60 \mathrm{~min}$ until $t=$ 0 , and tissues pretreated with PTX were treated for $30 \mathrm{~min}$ starting at $t=-90 \mathrm{~min}$. The increase of FLM adhesion during ischemia was attributed to accumulation of newly synthesized GAGs, for which PTX had no effect. With reperfusion, PTX inhibited shedding of GAGs significantly below control (preocclusion) values. Changes in red blood cell velocity $\left(V_{\mathrm{RBC}}\right)$ shown and for galactosaminoglycans $(C)$ and glucosaminoglycans $(D)$ reflect insignificant departures from control velocities, before and after the occlusion. Values are means \pm SD for 10 venules in each treatment. *Significantly different from control; ${ }^{\dagger}$ significant difference between occluded vessels with and without PTX.
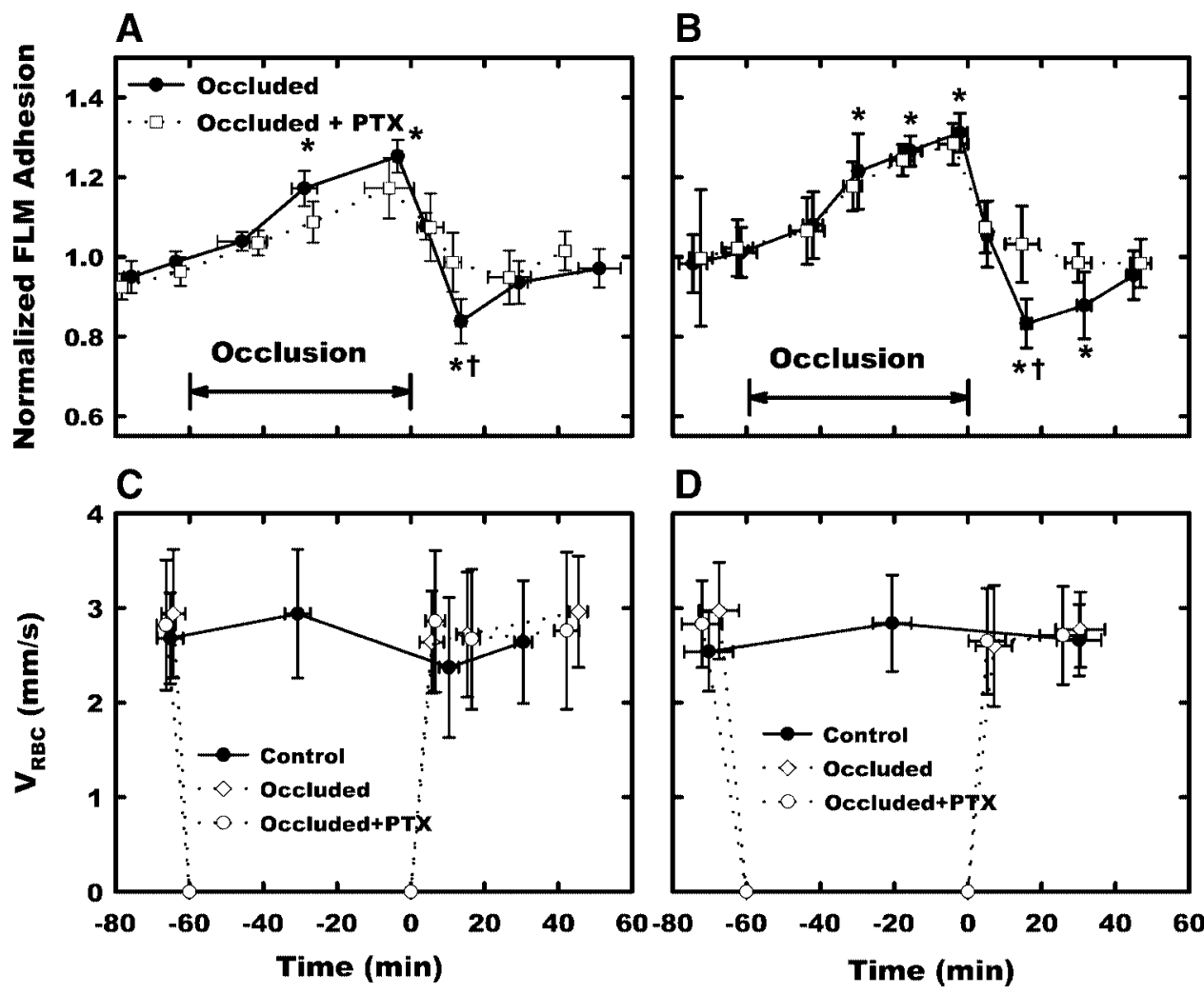

D

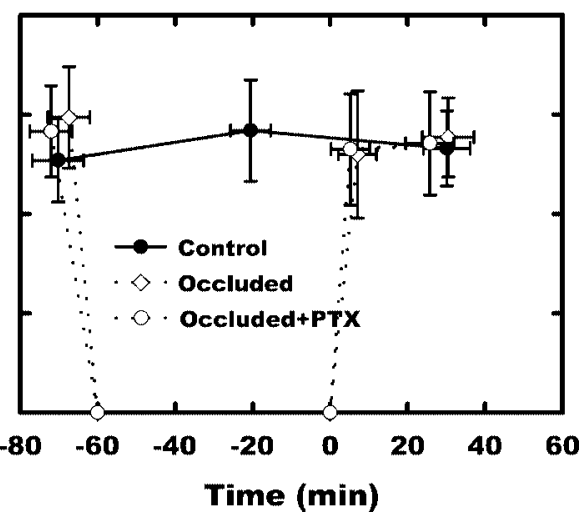

proteases, glycosidases, or lyases released or activated by $\mathrm{G}$ protein signaling. Plant lectins have been used previously to visualize components of the endothelial glycocalyx $(11,22$, $30)$. The lectin LEA has an affinity for the component $N$-acetyl glucosamine of HS and HA, and the lectin BS1 has an affinity for $N$-acetyl galactosamine of CS. Although it has been demonstrated that these lectins may bind to a broad spectrum of proteoglycans on the EC membrane (30), the apparent dominance of HS (14) and CS (26) suggests that the present data reflect the biosynthesis and shedding of these GAGs.

The significant decreases observed in BS1 FLM and LEA FLM adhesion after superfusion of fMLP (Fig. 1) suggest that fMLP initiates a mechanism that causes the rapid release of GAGs from the EC surface. The decrease in FLM adhesion does not appear to be related to the normal metabolic turnover of the GAGs because under control conditions the levels of FLM adhesion remain steady. Inhibition of $\mathrm{G}$ protein signaling with PTX shows that fMLP acts via G protein-sensitive receptors to initiate the loss of GAGs from the EC surface.

The chemotactic agent fMLP binds to the formylpeptide receptor (FPR), a GPCR located on both WBCs and ECs (27). The activity of the FPR has been shown to be sensitive to inhibition by PTX $(18,32)$. The ability of PTX treatment to inhibit fMLP-induced GAG shedding and WBC adhesion strongly suggests that the $G$ protein pathways begin an intracellular signaling mechanism that ultimately leads to shedding of the glycocalyx.

There are several physiological advantages to the rapid shedding of GAGs from the glycocalyx. A loss in GAG molecules enables WBC adhesion molecules greater access to their receptors. It has been shown that the glycocalyx provides steric hindrance of receptor and ligand binding during cell adhesion to the endothelium $(20,21,28)$ on the EC. Given that the molecular mass of syndecan-1 (160-400 kDa; Ref. 15) exceeds that of ICAM-1 (97-114 kDa; Ref. 4) and E-selectin (115 kDa; Ref. 4), shielding of these ligands by GAGs on the core protein could inhibit WBC-EC adhesion. In addition, freshly shed HS may become effector molecules that are loaded with factors such as thrombin and FGF, which may then be dispersed systemically.

Although the present data suggest that GAGs are shed from the glycocalyx, there is evidence that the core protein of the syndecan-1 proteoglycan is not (Fig. 3). The adhesion of H174 FLMs to the syndecan-1 core protein increased after superfusion with fMLP. The increase in adhesion is most likely due to the loss of the GAG molecules, which enables the H174 FLMs greater access to binding sites on the core protein. In the case of the fMLP stimulus, Western blots were performed on the effluent of an isolated and perfused segment of intestine, to probe for HS, CS, and syndecan-1 with appropriate antibodies. These studies revealed a time course of release of HS and CS that was consistent with in vivo observations of shedding and a negative response for the shedding of syndecan-1 (unpublished data). Previous studies found that expression of syndecan- 1 mRNA is reduced by the inflammatory agent TNF- $\alpha$ (15), but this change in protein expression takes much longer than the 60 min after fMLP superfusion observed here. Studies performed in vitro report that syndecan-1 is shed in response to thrombin and growth factors (34), but the difference in response may be due to the use of different tissues and treatments.

The use of lectin-labeled FLMs and lectins conjugated with FITC provides insight into the distribution of GAGs throughout the thickness of the glycocalyx and the ability of these 

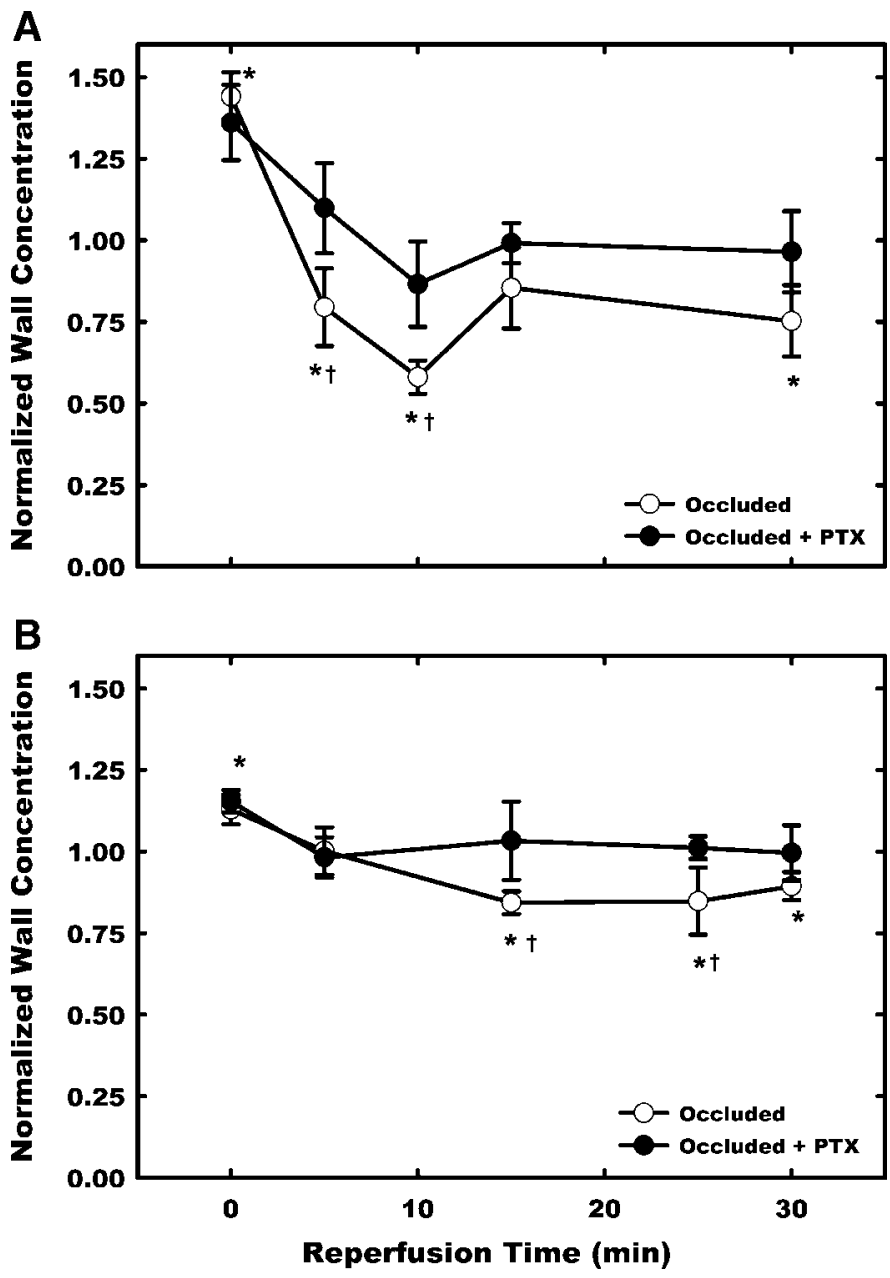

Fig. 7. Time course of FITC-labeled lectin binding to the EC during ischemia (60-min occlusion by a microprobe) followed by reperfusion. Binding of BS1-FITC to galactosaminoglycans $(A)$ and LEA-FITC to glucosaminoglycans $(B)$ within the glycocalyx was measured by confocal microscopy and normalized with respect to nonoccluded vessels in the same tissue. A blunted micropipette occluded proximal microvessels to produce ischemia from $t=$ -60 min to $t=0$. The tissues were fixed during reperfusion at the indicated times after release of the occlusion. Selected tissues were treated with PTX for $30 \mathrm{~min}$ starting at $t=-90 \mathrm{~min}$. Values are means $\pm \mathrm{SD}$ for 30 venules in each treatment. *Significantly different from control $(P<0.05)$; ${ }^{\dagger}$ occlusion response significantly different from occlusion with PTX pretreatment $(P<$ $0.05)$.

markers to penetrate the glycocalyx layer. Assuming that the lectins are globular in shape, a molecule of equal molecular mass $(\sim 120 \mathrm{kDa})$ would have a hydrodynamic diameter of $\sim 0.009 \mu \mathrm{m}$, which is roughly $1 / 10$ th that of the FLMs used $(0.1 \mu \mathrm{m})$. This dimension is comparable to the 8- to $10-\mathrm{nm}$ spaces determined by analysis of the periodic surface structure in electron micrographs (33) and the 5.7-nm pore exclusion size noted to limit glycocalyx permeation by dextrans (37). Thus permeation of the glycocalyx by FITC-labeled lectins is plausible, although it should be recognized that the relative penetration of LEA vs. BS-1 may be affected by differences in size and charge, as noted previously (37). The large microspheres are thus presumed to reflect the surface density of ligands on the glycocalyx.

The initial rates of GAG loss on the surface of the glycocalyx after onset of fMLP superfusion may be estimated from the initial slope of the BS1 FLM data for galactosaminoglycan $\left(-0.62 \mathrm{FLM} \cdot 100 \mu \mathrm{m}^{-1} \cdot \mathrm{min}^{-1}\right)$ and the LEA FLM data for glucosaminoglycan $\left(-1.41 \mathrm{FLM} \cdot 100 \mu \mathrm{m}^{-1} \cdot \mathrm{min}^{-1}\right)$ in Fig. 1. These rates of FLM loss with time represent a percentage of the reduction of the initial FLM counts per $100 \mu \mathrm{m}$ (before application of fMLP) equal to $-8.6 \% / \mathrm{min}$ and $-1.9 \% / \mathrm{min}$ for galactosaminoglycans and glucosaminoglycans, respectively. Across the depth of the glycocalyx, it appears from Fig. 5 that BS1-FITC also decreases at a rate $\left(0.32 \mathrm{ng} \cdot \mathrm{ml}^{-1} \cdot \mathrm{min}^{-1}\right.$ or $-4.8 \% / \mathrm{min})$ greater than that of LEA-FITC $(0.15$ $\mathrm{ng} \cdot \mathrm{ml}^{-1} \cdot \mathrm{min}^{-1}$ or $\left.-1.2 \% / \mathrm{min}\right)$. Hence, a greater percentage of galactosaminoglycans is lost in response to fMLP. Assuming equal rates of production and shedding of galactosaminoglycans and glucosaminoglycans by the EC, these rates are consistent with the $4: 1$ ratio of HS to CS within the glycocalyx, as noted previously $(14,26)$. That is, because the pool of glucosaminoglycans is four times greater than that of galactosaminoglycans, the same rate of net loss equates to a fourfold greater percentage loss for galactosaminoglycans. The much longer time for LEA-FITC to reach a minimum, compared with BS1-FITC (30 vs. $15 \mathrm{~min}$ in Fig. 5) may also reflect the substantially larger pool of glucosaminoglycans. The measurement of absolute concentrations of lectins sequestered on the EC (Fig. 5) may not reflect these proportions because of differences in the binding capacity of GAG molecules for their specific lectins.

The positive rate of accumulation of glucosaminoglycans and galactosaminoglycans during the ischemic period of Fig. 6, as evidenced by increased binding of BS1 FLMs and LEA FLMs, and the $35 \%$ and $15 \%$ increase in lectin binding to glucosaminoglycans and galactosaminoglycans, respectively (Fig. 7) suggest that in the absence of shearing forces, GAGs may grow longer or newly synthesized GAGs may accumulate in the glycocalyx. It has been shown that endothelial GAG synthesis increases after $24 \mathrm{~h}$ of high shear stresses (3). However, there is no prior evidence to suggest that abrupt changes in shear may rapidly affect their synthesis. Hence, the present results may reflect an invariant rate of synthesis that is offset by shear-dependent removal.

During reperfusion after 60 min of flow occlusion, venules experienced a significant loss in GAGs (Figs. 6 and 7). The rate of GAG loss during reperfusion followed a pattern similar to that established during fMLP superfusion. It is plausible that during the ischemic period there is a buildup of unbound GAGs in the glycocalyx that is convected away during reperfusion. The fact that the rate of loss during onset of reperfusion can be attenuated with PTX suggests that there is a G protein-mediated activation of proteases that cleave GAGs from proteoglycans (presumably mainly syndecan-1). The return of GAGs to preischemic levels under the influence of PTX may thus reflect shear removal of surplus GAGs, whereas the lower levels of GAG attained without PTX may reflect protease activation due to a change in shearing conditions. The rates of BS1 FLM loss (0.27 FLM/min; - 3.1\%), LEA FLM loss (0.48 FLM/min; $-2.5 \%)$, BS1 FITC loss $\left(1.24 \mathrm{ng} \cdot \mathrm{ml}^{-1} \cdot \mathrm{min}^{-1} ;-8.6 \%\right)$, and LEA-FITC loss $\left(0.54 \mathrm{ng} \cdot \mathrm{ml}^{-1} \cdot \mathrm{min}^{-1} ;-1.9 \%\right)$ were in ratios similar to those found during fMLP treatment.

On the basis of the decline from maximum to minimum levels of lectins attained in Fig. 7, it appears that $40 \%$ of the galactosaminoglycan and $25 \%$ of the glucosaminoglycan accumulated are removed by $\mathrm{G}$ protein-mediated shedding and 
the remainder is freely convected away by fluid shear. Numerous studies have linked shear to intracellular signaling via a PTX-sensitive mechanism (17). Prior studies of ischemiareperfusion injury indirectly measuring reductions in glycocalyx thickness during reperfusion have shown the ability of adenosine receptor activation to attenuate glycocalyx loss during reperfusion (22). In that study and the present study it is unlikely that the shedding results from reactive oxygen species, because constant superfusion of the tissues with Ringer solution precluded hypoxia during ischemia.

It is evident from the present studies that the glycocalyx is a dynamic entity that can be rapidly modulated by complex intracellular signaling systems within the EC and WBC. The present data suggest that individual GAG components of the glycocalyx may be shed by a $\mathrm{G}$ protein-sensitive mechanism in inflammation and ischemia-reperfusion. It is evident that GAG concentrations may be shear dependent and vary as rates of shedding and production are modulated by physiological conditions.

\section{ACKNOWLEDGMENTS}

The authors appreciate the technical assistance of Karren A. Trippett.

\section{GRANTS}

This work was supported in part by National Heart, Lung, and Blood Institute Grant HL-39286.

\section{REFERENCES}

1. Adamson RH. Permeability of frog mesenteric capillaries after partial pronase digestion of the endothelial glycocalyx. J Physiol 428: 1-13, 1990.

2. Adamson RH and Clough G. Plasma proteins modify the endothelial cell glycocalyx of frog mesenteric microvessels. J Physiol 445: 473-486, 1992.

3. Arisaka T, Mitsumata M, Kawasumi M, Tohjima T, Hirose S, and Yoshida Y. Effects of shear stress on glycosaminoglycan synthesis in vascular endothelial cells. Ann NY Acad Sci 748: 543-554, 1995.

4. Bevilacqua MP, Pober JS, Mendrick DL, Cotran RS, and Gimbrone MA Jr. Identification of an inducible endothelial-leukocyte adhesion molecule. Proc Natl Acad Sci USA 84: 9238-9242, 1987.

5. Colburn P, Kobayashi E, and Buonassisi V. Depleted level of heparan sulfate proteoglycan in the extracellular matrix of endothelial cell cultures exposed to endotoxin. J Cell Physiol 159: 121-130, 1994.

6. Constantinescu AA, Vink H, and Spaan JA. Elevated capillary tube hematocrit reflects degradation of endothelial cell glycocalyx by oxidized LDL. Am J Physiol Heart Circ Physiol 280: H1051-H1057, 2001.

7. Desjardins C and Duling BR. Heparinase treatment suggests a role for the endothelial cell glycocalyx in regulation of capillary hematocrit. Am J Physiol Heart Circ Physiol 258: H647-H654, 1990.

8. Dustin ML, Rothlein R, Bhan AK, Dinarello CA, and Springer TA. Induction by IL 1 and interferon- $\gamma$ : tissue distribution, biochemistry, and function of a natural adherence molecule (ICAM-1). J Immunol 137: 245-254, 1986.

9. Fitzgerald ML, Wang Z, Park PW, Murphy G, and Bernfield M. Shedding of syndecan-1 and -4 ectodomains is regulated by multiple signaling pathways and mediated by a TIMP-3-sensitive metalloproteinase. J Cell Biol 148: 811-824, 2000.

10. Gotte M, Joussen AM, Klein C, Andre P, Wagner DD, Hinkes MT, Kirchhof B, Adamis AP, and Bernfield M. Role of syndecan-1 in leukocyte-endothelial interactions in the ocular vasculature. Invest Ophthalmol Vis Sci 43: 1135-1141, 2002.

11. Henry CB and DeFouw DO. Differential lectin binding to microvascular endothelial glycoconjugates during normal angiogenesis in the chick chorioallantoic membrane. Microvasc Res 49: 201-211, 1995.

12. Henry CB and Duling BR. Permeation of the luminal capillary glycocalyx is determined by hyaluronan. Am J Physiol Heart Circ Physiol 277: H508-H514, 1999.

13. Ihrcke NS and Platt JL. Shedding of heparan sulfate proteoglycan by stimulated endothelial cells: evidence for proteolysis of cell-surface molecules. J Cell Physiol 168: 625-637, 1996.
14. Ihrcke NS, Wrenshall LE, Lindman BJ, and Platt JL. Role of heparan sulfate in immune system-blood vessel interactions. Immunol Today 14: 500-505, 1993.

15. Kainulainen V, Nelimarkka L, Jarvelainen H, Laato M, Jalkanen M, and Elenius K. Suppression of syndecan-1 expression in endothelial cells by tumor necrosis factor- $\alpha$. J Biol Chem 271: 18759-18766, 1996.

16. Kato M, Wang H, Bernfield M, Gallagher JT, and Turnbull JE. Cell surface syndecan-1 on distinct cell types differs in fine structure and ligand binding of its heparan sulfate chains. J Biol Chem 269: 18881-18890, 1994.

17. Labrador V, Chen KD, Li YS, Muller S, Stoltz JF, and Chien S. Interactions of mechanotransduction pathways. Biorheology 40: 47-52, 2003.

18. Lad PM, Olson CV, and Smiley PA. Association of the N-formyl-MetLeu-Phe receptor in human neutrophils with a GTP-binding protein sensitive to pertussis toxin. Proc Natl Acad Sci USA 82: 869-873, 1985.

19. Lipowsky HH and Zweifach BW. Application of the "two-slit" photometric technique to the measurement of microvascular volumetric flow rates. Microvasc Res 15: 93-101, 1978.

20. Mulivor AW and Lipowsky HH. Role of glycocalyx in leukocyteendothelial cell adhesion. Am J Physiol Heart Circ Physiol 283: H1282H1291, 2002.

21. Patel KD, Nollert MU, and McEver RP. P-selectin must extend a sufficient length from the plasma membrane to mediate rolling of neutrophils. J Cell Biol 131: 1893-1902, 1995.

22. Platts SH, Linden J, and Duling BR. Rapid modification of the glycocalyx caused by ischemia-reperfusion is inhibited by adenosine A2A receptor activation. Am J Physiol Heart Circ Physiol 284: H2360-H2367, 2003.

23. Porter GA, Palade GE, and Milici AJ. Differential binding of the lectins Griffonia simplicifolia I and Lycopersicon esculentum to microvascular endothelium: organ-specific localization and partial glycoprotein characterization. Eur J Cell Biol 51: 85-95, 1990.

24. Pries AR, Secomb TW, and Gaehtgens P. The endothelial surface layer. Pflügers Arch 440: 653-666, 2000.

25. Pries AR, Secomb TW, Jacobs H, Sperandio M, Osterloh K, and Gaehtgens P. Microvascular blood flow resistance: role of endothelial surface layer. Am J Physiol Heart Circ Physiol 273: H2272-H2279, 1997.

26. Rapraeger A, Jalkanen M, Endo E, Koda J, and Bernfield M. The cell surface proteoglycan from mouse mammary epithelial cells bears chondroitin sulfate and heparan sulfate glycosaminoglycans. J Biol Chem 260: 11046-11052, 1985.

27. Rotrosen D, Malech HL, and Gallin JI. Formyl peptide leukocyte chemoattractant uptake and release by cultured human umbilical vein endothelial cells. J Immunol 139: 3034-3040, 1987.

28. Sabri S, Solar M, Foa C, Poierres A, Benoliel A, and Bongrand P. Glycocalyx modulation is a physiological means of regulating cell adhesion. J Cell Sci 113: 1589-1600, 2000.

29. Saunders S, Jalkanen M, O'Farrell S, and Bernfield M. Molecular cloning of syndecan, an integral membrane proteoglycan. J Cell Biol 108: 1547-1556, 1989.

30. Schnitzer JE, Shen CPJ, and Palade GE. Lectin analysis of common glycoproteins detected on the surface of continuous microvascular endothelium in situ and in culture: identification of sialoglycoproteins. Eur J Cell Biol 52: 242-251, 1990.

31. Sorrenson J, Bjornson A, Ohlson M, Ballermann BJ, and Haraldsson B. Synthesis of sulfated proteoglycans by bovine glomerular endothelial cells in culture. Am J Physiol Renal Physiol 284: F373-F380, 2003.

32. Spangrude GJ, Sacchi F, Hill HR, Van Epps DE, and Daynes RA. Inhibition of lymphocyte and neutrophil chemotaxis by pertussis toxin. J Immunol 135: 4135-4143, 1985.

33. Squire JM, Chew M, Nneji G, Neal C, Barry J, and Michel C. Quasiperiodic substructure in the microvessel endothelial glycocalyx: a possible explanation for molecular filtering? J Struct Biol 136: 239-255, 2001.

34. Subramanian SV, Fitzgerald ML, and Bernfield M. Regulated shedding of syndecan- 1 and -4 ectodomains by thrombin and growth factor receptor activation. J Biol Chem 272: 14713-14720. 1997.

35. Van den Berg BM and Vink H. The endothelial glycocalyx protects against myocardial edema. Circ Res 92: 592-594, 2003.

36. Vink $\mathbf{H}$ and Duling BR. Identification of distinct luminal domains for macromolecules, erythrocytes, and leukocytes within mammalian capillaries. Circ Res 79: 581-589, 1996.

37. Vink H and Duling BR. Capillary endothelial surface layer selectively induces plasma solute distribution volume. Am J Physiol Heart Circ Physiol 278: H285-H289, 2000. 